\title{
Deducing a mechanism of all musculoskeletal injuries
}

\author{
Geoffrey Verrall ${ }^{1}$ \\ Bronwyn Dolman²,3 \\ 1 Department of Sports Medicine, South Australian \\ Institute, Adelaide, Australia \\ 2 ATRAD Pty Ltd, Thebarton, Australia \\ 3 Department of Physical Sciences, University of \\ Adelaide, Australia
}

Corresponding author:

Geoffrey Verrall

Department of Sports Medicine, South Australian

Institute

27 Valetta Rd, Kidman Park

5025 Adelaide, Australia

E-mail: verrallg@bigpond.com

\section{Summary}

Background: The mechanism of musculoskeletal (MSK) injuries is not well understood. This research applies principles of elastic motion to the anatomy and movement patterns of MSK structures. From this an insight into the application and timing of forces on MSK structures can be established and the mechanism/s of injury derived.

Methods (Current Knowledge): All MSK structures demonstrate varying degrees of elasticity. Movement occurs primarily as a consequence of Muscle Tendon Unit (MTU) shortening. The application of an applied external force results in MSK structure lengthening.

Results: The MTU acts as a non-idealised Hookean Spring. The resting length of MSK structures is the minimum distance between attachment points. The anatomical constraints results in MSK structures having adequate compressive strength during shortening. Thus MSK injuries only occur during lengthening of the MSK structure. From this with knowledge of MSK movement cycles, we can derive the mechanism of injury.

Conclusions: MSK injuries result from an inability to counter applied forces whilst lengthening. Muscles, tendons and ligaments can only injure during their lengthening contraction phase. Insertional tendons and bone near attachment points injure during the MTU shortening phase. Injuries to other MSK structures can occur independent of the lengthening and shortening phases such as direct contact injuries.
KEY WORDS: insertional tendon, muscles, stress fracture, tendons, tensile force.

\section{Introduction}

Musculoskeletal (MSK) injuries are a common presentation in clinical practice, not limited to athletic populations. The morbidity associated with MSK injuries places a significant burden on health care resources ${ }^{1}$. The unknown causality of MSK injuries results in many different modes of treatment which are often non-specific, and lack scientific evidence for their use ${ }^{2}$. Clinical studies on MSK injuries have focused on identifying risk factors for specific injuries with limited understanding of the injury mechanism $(s)^{3}$. Understanding MSK injury mechanism(s) would allow us to identify the causality of these injuries enabling the development of a more efficient use of health system resources in the areas of diagnosis, treatment and rehabilitation.

Recent physiological and biological studies incorporating the elastic nature of the muscle fibers and tendons ${ }^{4-8}$ have advanced the understanding of musculoskeletal movement. Knowledge of the lengthening and shortening movement patterns of these elastic structures has also advanced ${ }^{9-12}$. However, modeling and in situ measurements of MSK function are inherently difficult or impossible being limited, in part, by current technology. Therefore, the aim of this research is not to conduct further experimentation, but to analyze and combine previous research in order to derive further insight into the forces associated with the movement patterns relevant to MSK structures.

\section{Methods}

Anatomical components of the MSK system are well understood and include the Muscle Tendon Unit/s (MTU/s) and Ligament Bone Unit/s (LBU/s). Although these units vary considerably in anatomical configuration, they have a relatively uniform macro-structure (Fig. 1). By virtue of the fixed position of the MTU and LBU attachment points on the skeleton even with three dimensional movements there exists a minimum and maximum separation for each of these units. Thus MSK structures are therefore constrained by their anatomical design, and operation outside of this design will only occur in catastrophic injuries such as those associated with significant bone fractures. 


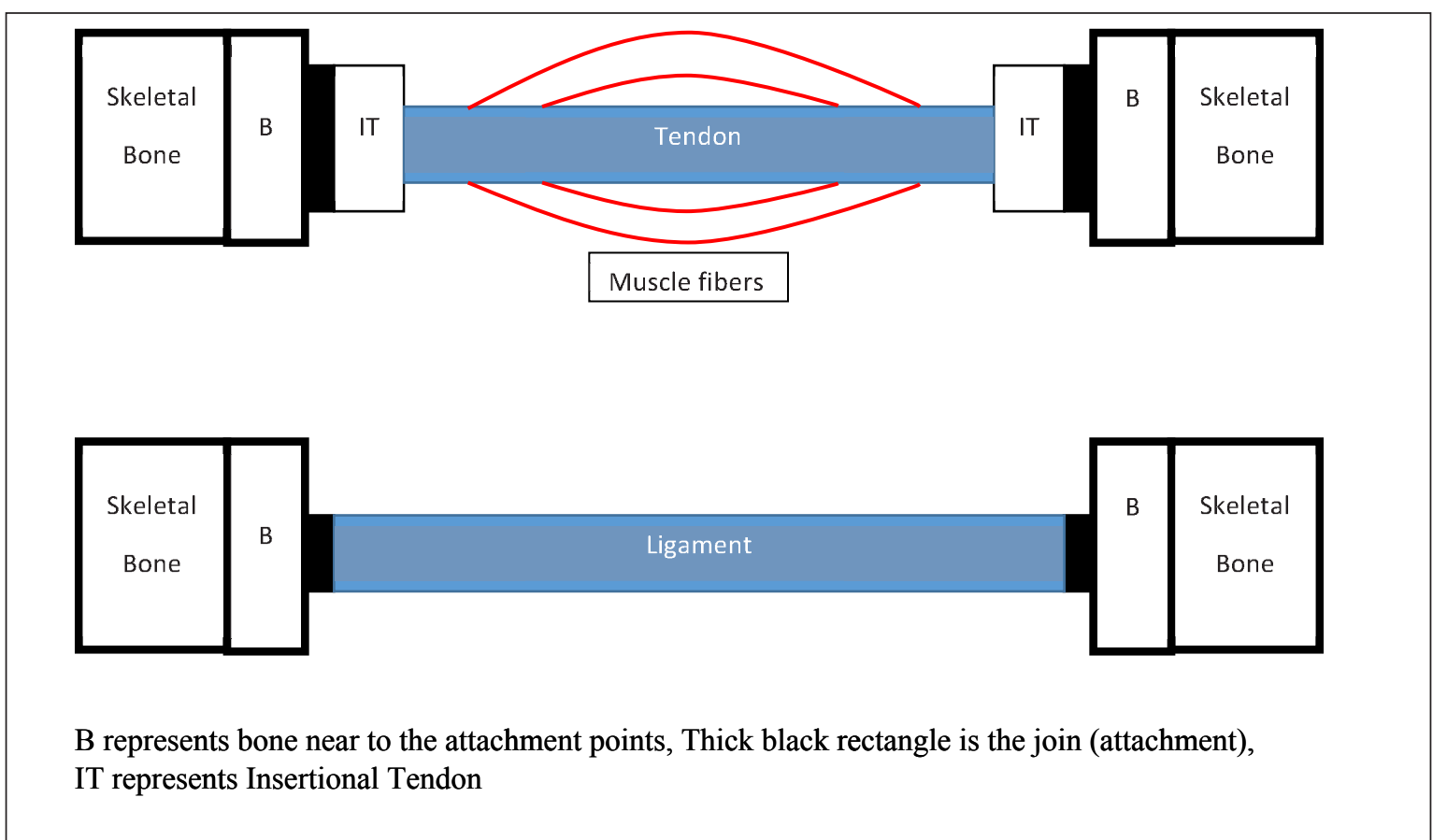

Figure 1. Schematic diagram of anatomy and structure of the musculoskeletal system.

Previous research demonstrates all structures of the musculoskeletal system display varying degrees of elasticity $5,7,13$ where elasticity is defined as the ability of a solid material to return to its original shape following deformation ${ }^{14}$. The role of the elastic structures of the MSK system is both to absorb applied forces $^{7,15}$, and to act on the skeleton to produce movement ${ }^{8}$. Movement is considered to be primarily the role of the more elastic, compared to other MSK structures, MTU's ${ }^{16}$.

Musculoskeletal movement is accomplished by MTU shortening 7,15,17 applying force to the skeleton via the attachment points. The forces associated with the shortening movement, including ground contact, need to be absorbed by other elastic MSK structures. This occurs by the MSK structures lengthening to minimize the impact on bones and MSK structures $8,15,18$. Thus each MTU lengthens in response to an applied force, and subsequently shorten to produce movement.

The process of MTU lengthening and shortening is known as the stretch-shortening or contraction cy$\mathrm{cle}^{19}$. When the MTU is at its minimum length, that is the distance between proximal and distal skeleton attachment points is minimal, the tendons of the MTU are folded (termed crimps) ${ }^{20}$ and the tendon is slightly longer than the minimum distance between the attachment points, termed the tendon-slack length ${ }^{20}$. In a lengthening contraction, the crimps first unfold and the slack length is taken up, which is then followed by lengthening of the tendon and muscle fibers ${ }^{20}$. Muscle fibers are then actively recruited and lengthen to match force requirements 8,15 . Active lengthening refers to the binding, release, and rebinding of the muscle fibers, known as the sliding filament theory ${ }^{19}$, with this process requiring significant metabolic energy $^{20}$.

When a MTU has reached its maximum required length, the unit has attained elastic potential energy, which is subsequently converted to kinetic energy as shortening commences ${ }^{8,21}$. MTU shortening is a more powerful contraction ${ }^{4}$, as it occurs over a shorter time period when compared to lengthening ${ }^{8}$, with forces decreasing rapidly as the MTU shortens ${ }^{4}$.

Recent research demonstrates the MTU can alter its stiffness in response to an applied force ${ }^{5,7}$, where stiffness is defined as the extent to which an object can resist deformation under force application ${ }^{15}$. This response occurs automatically without nervous system input ${ }^{4}$. The converse is also true, as force decreases with shortening the MTU becomes more compliant ${ }^{7}$. Variations in stiffness occur on very short time scales, within a single contraction cycle. The implications of this ability are not yet fully understood $^{8}$. Despite this complexity recent studies demonstrate a predictable force-length relationship ${ }^{4,6}$, with force on the MTU being proportional, albeit non-linear, to displacement.

In contrast to the MTU, the LBU and other MSK structures lengthen and shorten to varying degrees dependent on their elastic properties with these process being considered mostly passive, that is without a significant metabolic energy requirement. We will refer to these non-MTU structures as having a contraction cycle notwithstanding the above significant difference to the MTU contraction cycle. LBU's demonstrate some ability to store elastic energy 22 and other musculoskeletal structures such as bones and ligaments demonstrate an ability to vary stiffness over longer time frames in response to training ${ }^{23,24}$ and aging ${ }^{25}$. 
This illustrates the dynamic elasticity of all components of the MSK system.

The analysis undertaken in this research meets the ethical standards of this journal ${ }^{26}$.

\section{Results}

The action of the MTU with repetitive lengthening and shortening fits the description of being spring-like with this having been postulated in several previous studies $4-8,12,21$. Traditional springs obey Hooke's Law, which states the force on an elastic structure is proportional to the displacement of that structure from its resting length. In other words, when an elastic structure is at its resting length, it is neither compressed or stretched, and experiences no force ${ }^{14}$. Displacement from its resting length through either compression or stretching, results in the structure experiencing a force in the opposing direction, acting to restore the structure to its resting length. The greater the structure is displaced from its resting length the higher the restoring force will be as force is proportional to displacement. Additionally, Hooke's Law equates with a spring constant, $k$, which describes the stiffness of the elastic structure. Stiffer materials have larger $k$ values.

To derive forces on MSK structures using elastic motion principles we must establish the case of the MTU spring defining both the resting length and spring constant. Studies on the MTU demonstrate forces will either increase or decrease with unidirectional displacement. Lengthening is achieved through the action of forces generated externally to the MTU effectively pulling the MTU to its maximal length. Shortening is then achieved by the restoring force acting on the MTU to return it to its minimal length. If the resting length was at a non-minimal or non-maximal length, forces in the MTU would decrease as the muscle approached resting length, become zero at resting length, and then increase as the MTU continued to contract away from resting length. This concept is neither logical nor demonstrated by multiple MTU experiments. Thus the resting length must be at either the minimal or maximal distance between the two attachment points. A previous study defined resting length where there was no strain in the MTU21. Further, it is understood the tendon and ligament are slack at minimal length, corresponding to minimal force ${ }^{17,21}$, and thus the resting length for the MTU, and the LBU, must be at the minimal displacement between skeleton attachment points. The concept of the elastic motion resting length can be distinguished from the position when the muscles are not moving and as such are at rest. MTU at anatomical rest refers to the muscle tone of the MTU that has resting tension to help stabilize the position of the bones and joints but is not contracting. At anatomical rest the MTU's need to have some ability to alter length to enable the initiation of movement when required. If we consider an MTU to be at resting length in the anatomical rest position it would have no ability to shorten, and hence move the skeleton. Similarly, an opposing MTU will be at maximal stretch, and thus the only movement available to the skeleton is for the stretched MTU to shorten, in turn lengthening the MTU at rest. This system would be highly inefficient, and thus the anatomical rest position is not the resting length.

For the case of the MTU, k cannot be considered constant, as the MTU has the ability to increase stiffness during lengthening, and decrease stiffness, becoming more compliant, during shortening. Thus it is considered the MTU has a responsive (non-constant) spring constant. As it has been demonstrated in laboratory studies, the response in spring constant always acts in the same direction as displacement from resting length, becoming stiffer with lengthening and more compliant with shortening. Thus despite potential non-linear changes in $k$, forces in the MTU will still increase with lengthening and decrease with shortening, and we can consider the MTU acts as a non-idealized Hookean spring.

With a traditional spring $\mathrm{k}$ is a constant and describes the stiffness of the material. As the $\mathrm{k}$ is not constant in the case of the MTU we will term this variation in stiffness the responsive spring constant k. For a contraction cycle, consider first the simplest case of a cycle at a fixed minimal load with no variation in MTU stiffness, such that $\mathrm{k}_{\mathrm{r}}$ can be approximated by the constant k. Hooke's Law then dictates the force on the MTU will increase with increasing displacement, and decrease as the MTU returns to its minimal resting length. This simple model makes conceptual sense, stating forces on the muscle are highest when the MTU is at its maximal stretch, and minimal when the MTU is at resting length.

If we now consider the case of dynamic $\mathrm{k}_{\mathrm{r}}$ within the lengthening contraction, the concept of maximal force at maximal displacement must still apply. To examine this statement, consider the alternative of maximal force not occurring at maximal displacement. For this to occur, $\mathrm{k}_{\mathrm{r}}$ must decrease by a quanta equal to or greater than the displacement, which implies the MTU has become more elastic or slack while lengthening. By analogy, consider a spring or rubber band becoming more elastic as it is stretched. When this spring or rubber band is released it would have less potential energy available to return to its resting length than if the spring constant remained constant or increased. Logic therefore dictates the $\mathrm{k}_{\mathrm{r}}$ must either remain constant or increase with lengthening, which is supported by studies demonstrating a higher $k$ value (stiffness) of the MTU with increasing lengthening force application ${ }^{4,6}$.

Applying the same argument in reverse, as the MTU returns to its minimal resting length, where it is known the tendon is slack, the spring constant must remain constant or decrease. If the MTU was stiffening as it shortened, that is $\mathrm{k}_{\mathrm{r}}$ increasing, the tendon could not return to slack. This is demonstrated in studies which show MTU stiffness increasing with lengthening and decreasing with shortening ${ }^{4}$.

We can therefore state that despite variations in the 
spring constant of a typical MTU, it will only act in the same direction as the displacement, and thus forces on the MTU increase with lengthening, are maximal at maximal displacement, and decrease with shortening to be minimal at minimal displacement.

Other, non-MTU, elastic musculoskeletal structures have not demonstrated, as far as can be currently measured, an ability to alter stiffness in a short time frame but they still undergo elastic lengthening and shortening.

Clinical studies have demonstrated movement-related injury can occur to all elastic MSK structures (muscle fibers, tendons, ligaments, bones and joints). Injuries occur in circumstances where elastic structures are unable to counter an applied force ${ }^{27}$. In order to operate without injury, MSK structures when lengthening require adequate tensile strength, with strength defined as the force per unit area the structure can generate ${ }^{14}$. In contrast MSK structures when shortening will require adequate compressive strength in order to operate without injury (Fig. 2). Thus under normal, uninjured, operation MSK structures will have adequate compressive and tensile strength.

We will specifically analyze injuries that occur within the anatomical constraints of a minimum and maximum distance between attachment points on the skeleton, an example being muscle strain injury. Obviously injuries can occur by exceeding this constrained distance, an example being a bone fracture. Within normal contraction cycle (lengthening-shortening) operation, the anatomical constraints of the human skeleton dictate the minimum distance between the skeleton attachment points cannot be exceeded. Thus MSK structures cannot be shortened beyond this minimum, which corresponds to our defined resting length. Consequently, MSK structures do not ex- perience compressive forces beyond restorative forces with contraction to resting length. Thus the MSK structures, operating within our definition of a constrained anatomical system, will have adequate compressive strength.

We define failure as broadly referring to all changes within a MSK structure that may lead to injury. If structure failure occurs during lengthening, further lengthening will only act to increase tensile force on this elastic structure whereas failure during shortening result in decreased compressive forces as the unit continues to shorten. Thus although we cannot determine injury timing and consequently etiology from this analysis we can categorically state irrespective of the timing of unit failure, for injuries associated with musculoskeletal elastic lengthening and shortening, it will be only in lengthening, where forces are increasing, that failure will cause an injury (Fig. 3). Thus constructing specific musculoskeletal injury mechanisms only requires knowledge of when the respective MSK structure is lengthening.

Muscle fibers, tendons and ligaments have a well understood lengthening and shortening cycle and are at risk for injury during their respective lengthening contractions.

For the insertional tendon (a specialized structure with transition from tendon to highly mineralized bone) and nearby bone structures we will abbreviate as IT-B. The IT-B are lengthened from applied forces pulling the attachment points apart with resulting in MTU lengthening. IT-B are also lengthened from the subsequent MTU shortening contraction which pulls the attachment points together. An elastic structure cannot be lengthened infinitum and must attain resting length. This occurs where there is minimal force on the structure. This occurs at the end of MTU shortening where there is minimal pull on the attachment

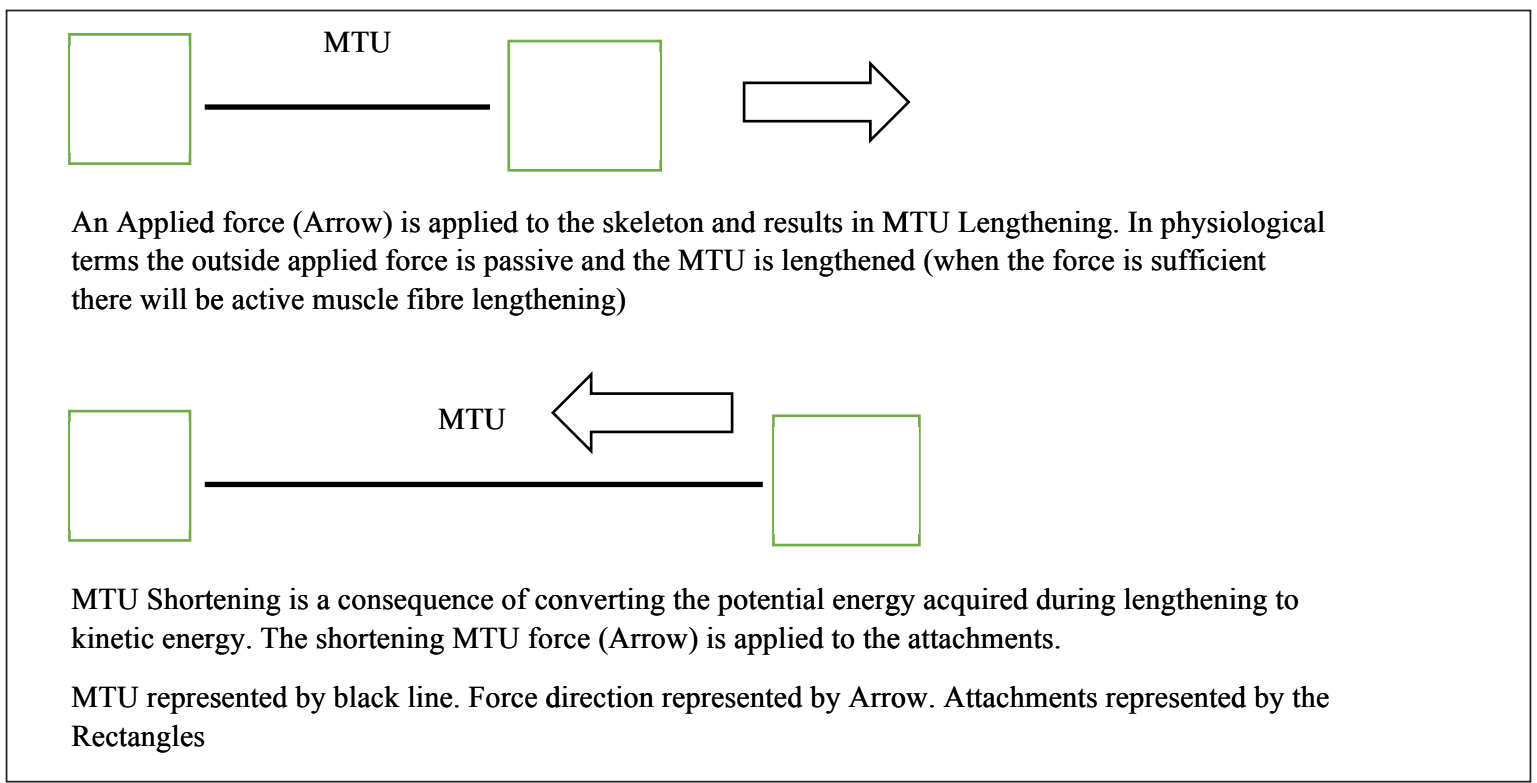

Figure 2. MTU lengthening and shortening applied forces. 


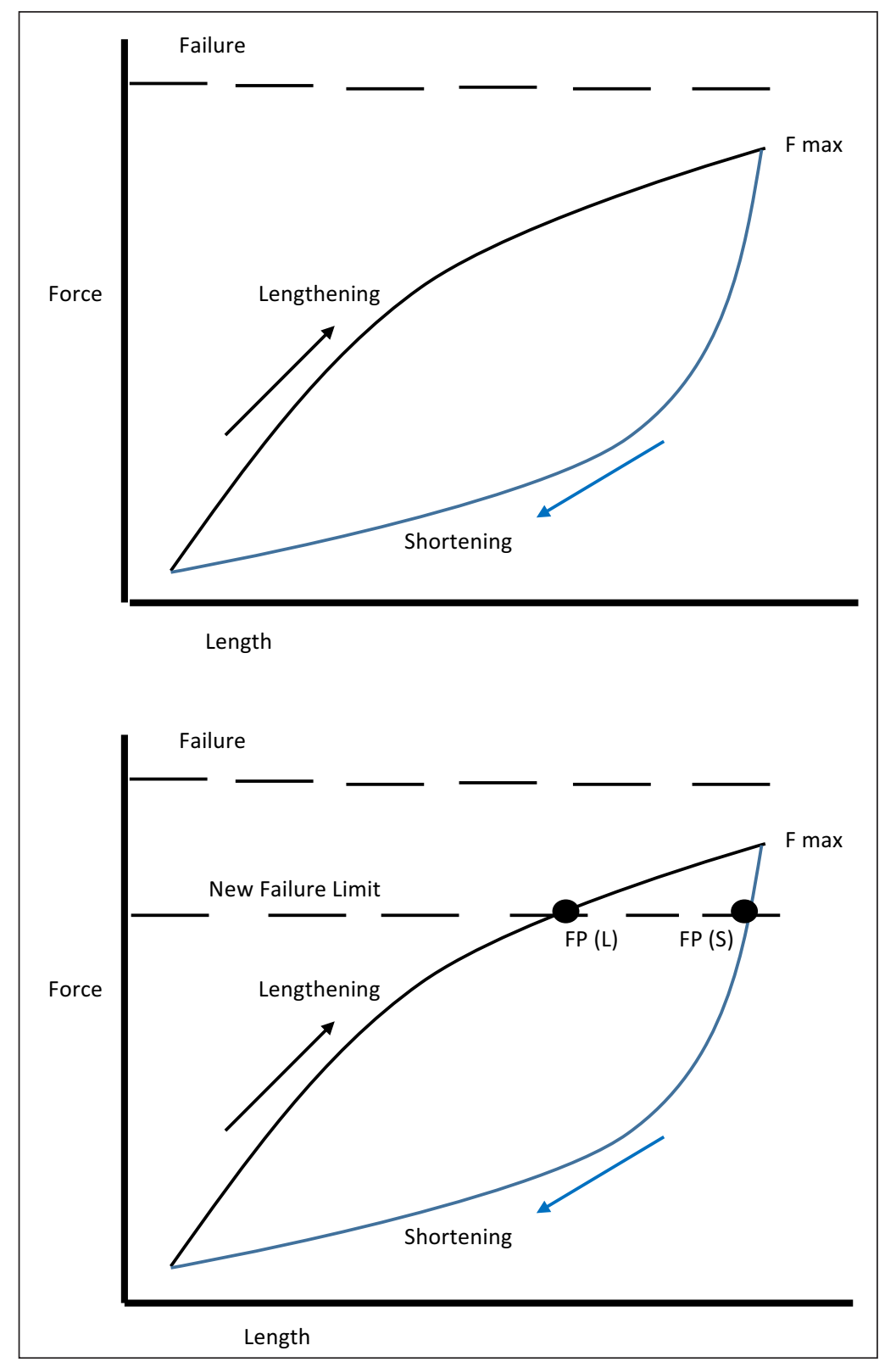

Figure 3. Force length curve of lengthening and shortening.

Assume Failure Limit decreases as demonstrated in the diagram. Failure point could conceivably occur in lengthening or shortening. During lengthening if Failure Point $\mathrm{FP}(\mathrm{L})$ occurs the force will continue to increase with further lengthening resulting in breeching the failure limit with subsequent injury. If failure occurs during shortening $\mathrm{FP}(\mathrm{S})$ the force will continue to decrease with the failure limit not being breeched thus no injury will occur.

An important concept in Figure 3 demonstrates that although there may be an alteration in the timing of the injury with regards to failure limit occurring it is only in lengthening that force exceeds this failure limit with a subsequent injury.

points from both the contracting/shortening unit and the applied external forces. In contrast at the end of MTU lengthening with increasing stiffness the unit will have increased tension, which will in effect try to pull the attachment points together, and thus the IT-B is prevented from attaining resting length. Overall this model proposes that all MSK structures will attain resting length, and therefore force will be at a minimum, simultaneously at the end of MTU shortening, optimally placing all structures to again begin lengthening and absorb forces.

We consider that it is with MTU shortening that this is the time for IT-B lengthening injury. The reasons for this is that with MTU shortening the force is directly applied to the IT-B. This contrasts to when externally applied force is applied to the IT-B as it is indirectly applied as it attenuated by other structures prior to
IT-B application. Also MTU shortening force is more powerful (force applied over a shorter time period) than those experienced with MTU lengthening. Finally, with the application of external force the more elastic muscle fibers and tendons will lengthen thereby also attenuating the force applied to the IT-B (Fig. 4). A similar argument is presented for the LBU attachment point structures but with a significantly reduced ability of the LBU to store elastic potential energy and subsequently less power with subsequent shortening, injuries at the LBU attachment sites are uncommon.

Injuries are also associated with externally applied forces that are independent of the contraction cycle of MSK structures. Most fractures are considered to be a consequence of tensile (lengthening) forces but there are some fractures that are compressive (short- 
For simplicity we will look at the force displacement diagram from one attachment point.

Force is represented by unfilled arrows, Lengthening is represented by filled arrow pointing to right of page, Shortening is represented by filled arrow pointing to the Left of the page.

Beginning of Lengthening: An External Force (EF) (unfilled arrow) is applied from outside the Muscle Tendon Unit (MTU). EF is due to another MTU shortening and/or ground reaction force and/or other applied external force. This EF is applied to the MTU after being attenuated somewhat by the skeleton and other joins of other muscle tendon units.
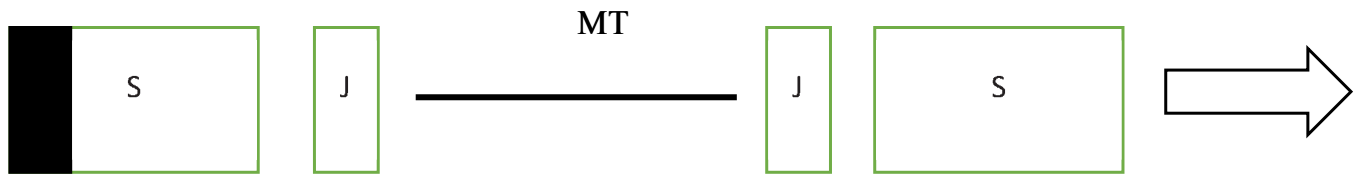

$\mathrm{S}=$ Skeletal Bone, $\mathrm{J}=\mathrm{J}$ oin consisting of the IT-B. Insertional tendon $(\mathrm{T})$ on the MT side and the nearby bone (B) on the skeleton side of the join (see Figure 1), MT=Muscle tendon, Filled Block is the skeleton body anchor

Early Lengthening: Force is EF (lengthening the unit) and Restoration Force (RF) (trying to shorten the $\mathrm{MT}$ ). Overall the force EF $>$ RF so lengthening to the skeleton, the IT-B and the MT continues.
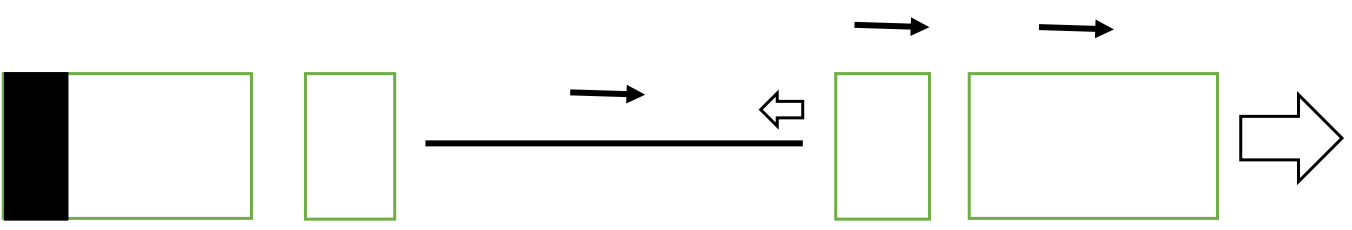

End of Lengthening: The EF drops to zero and the MT RF (large unfilled arrow) increases. There is alos an increase in dynamic tension (small unfilled arrow). The MTU is at its maximum length with no movement of MT and IT-B.
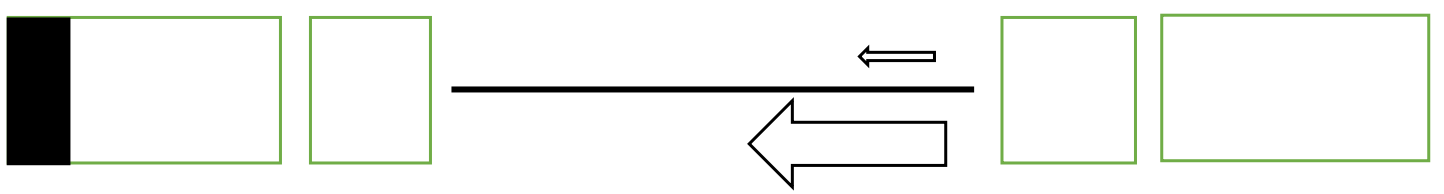

Figure 4. Force and lengthening/shortening diagram (continue $\rightarrow$ )

ening) in nature. The other obvious injuries independent of the contraction cycle are direct contact injuries to the surfaces of joints and contusions (bruises) of muscles and other musculoskeletal structures. A power point with animation of the basic theory is available here. https://drive.google.com/file/d/0By91_ RcMoIYaODB4VzJnWTM3VWc/view?usp=sharing

\section{Discussion}

This research leads us to propose a Mechanism Of all Musculoskeletal Injuries (MOMI) with injuries to all musculoskeletal structures resulting from an inability to counter applied forces whilst the structure is lengthening. The elegance of the MOMI is the simplicity by which musculoskeletal injury mechanisms can be described. This should lead to an improved ability to determine injury etiology, improve diagnosis capabilities, and enable the design and implementation of more targeted treatment regimes. As injury occurs to muscle fibers, tendons and ligaments on lengthening a potential treatment or prevention strategy is to train these structures to withstand higher forces during lengthening, or alternatively diminish the force of the externally applied forces that bring about lengthening. The same principle applies to insertional tendons and nearby bone (stress fractures) 
Beginning of Shortening: MT RF (large unfilled arrow) acts to shorten the muscle tendon unit. The EF is minimal (small unfilled arrow) from a passive weight of skeleton. The IT-B, not being as elastic as the MT, gets pulled and continues to lengthen (filled arrows to right of page). The MT restoration force is directly applied and is more powerful (force applied over a shorter time span) than lengthening applied EF.
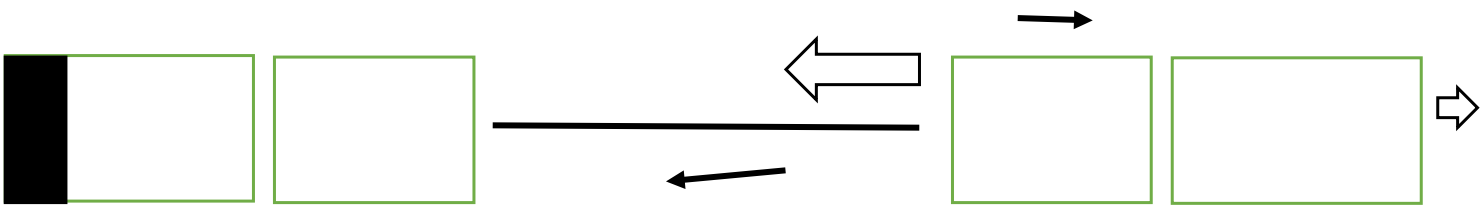

Late shortening: The MT RF decreases (larger unfilled arrow) with the EF (smaller unfilled arrow) being unchanged. This force results in continuing MT shortening (filled arrow) as the restoring force of the MT is larger than the EF. As the RF of the IT-B and skeleton shortens is greater than the RF of the MT shortening and EF (unfilled arrows) the IT-B and skeleton will now shorten.

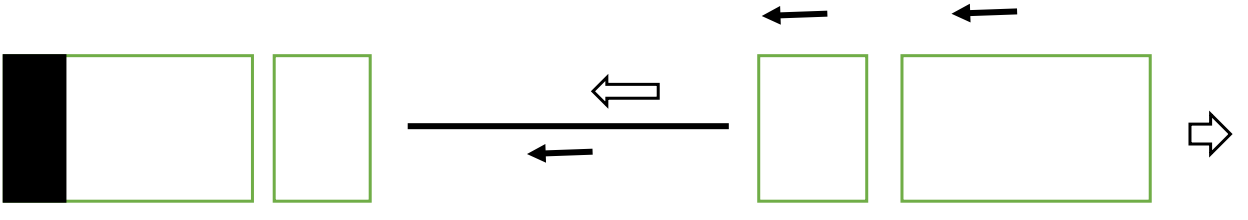

End of Shortening: There is minimal force application from either EF or MT RF and the structure has tension at its minimum. All structures MT, IT-B and skeleton are at their minimal (physical) resting lengths
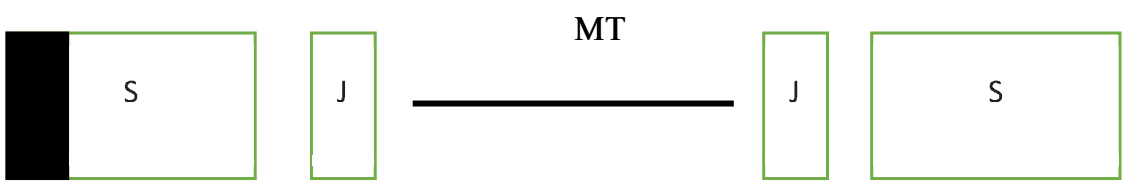

Figure 4. (continue)

injuries where either the force of the MTU shortening needs to be reduced or the structure strengthened. Science is about hypothesis and testing this hypothesis. Proof or disproof of the MOMI will likely follow by exception, both as an overarching theory, or for all individual common MSK injuries. Proof is currently not possible due to, but not limited to, the inability to perform in vivo human tests, the lack of a suitable animal model, the lack of a suitable laboratory model for living tissues and the anatomical complexity. The inability to measure small movements and forces in vivo is also a major issue. Consider the typical biceps femoris MTU which has been demonstrated to stretch $9.5 \%$ of its original length 28 . If the attaching structures are 200 times less elastic, for example bone compared to tendon ${ }^{29-31}$, this results in a $0.48 \%$ increase in original length. The insertional tendon transition mineralized zone occurs over $1 \mathrm{~mm}$ thus movements of $0.048 \mathrm{~mm}$ (48 micron) would need to be measured. Opposition to this analysis will occur in three areas. Firstly, the simplicity of our conclusion will challenge many existing paradigms in MSK injuries. Recent improvements in the understanding of MTU contraction has enabled us to apply elastic motion principles in order to derive forces on MSK structures. Secondly the non-linearity of force displacement during lengthening and shortening will cause a non-acceptance of those who fail to comprehend our analysis, of a non- 
idealized Hookean spring. Thirdly there is the argument that the elastic MSK structures are actually viscoelastic structures. An elastic MSK viscoelastic material will return to its original shape after any deforming force has been removed (i.e., it will show an elastic response) even though it will take time to do so (i.e., it will have a viscous component to the response). Thus we believe using our defined model of lengthening and shortening to attain the property of the movement components of the MSK system acting as non-idealised Hookean spring is justified. Viscoelasticity is discussed in Appendix 1.

\section{Conclusion}

Injuries for all musculoskeletal structures result from an inability to counter applied forces whilst the structure is lengthening. Injury during shortening would only occur if the unit had inadequate compressive strength, which we have demonstrated is prevented by anatomical constraints during normal operation. Injuries to the more elastic structures (muscle fibers, tendons and ligaments) will only occur during their respective lengthening phases. In contrast, the less elastic attachment point structures, insertional tendon and bone near to the attachment, injure with lengthening which is a consequence of the force applied during muscle tendon unit shortening. Exceptions to these principles occur with direct contact injuries, such as those that occur to joints.

\section{Appendix 1. Elasticity and Viscoelasticity}

All biological structures to some extent but particularly the more elastic muscle fibres and tendons are considered to have the property of viscoelasticity.

By further explanation the key viscoelastic properties exhibited by muscle fibers and tendons are; 1) nonlinear response of force and displacement, 2) Hysteresis with force plotted against displacement being different for stretch and recoil, 3) Velocity dependent stiffness, 4) Creep (with a constant force length increases with time), 5) Load relaxation.

The non-linear force-displacement response is well known but recent work demonstrates a predictable response. Hysteresis can be explained by the property of being able to alter stiffness in response to required force. A recent study may demonstrate a reason for this observation ${ }^{32}$. Velocity dependent stiffness can be explained by the order in which the lengthening occurs - crimp, then passive tendon then active muscle fibre activation with the muscle fibers being able to alter stiffness to required force. Creep almost certainly reflects biological properties that differ from inert structures leading to some plasticity, but still predictable force displacement relationship, of the MTU. Finally, if a structure is held at length it has acquired significant potential energy, if it does not convert this to shortening kinetic energy then this energy needs to be dissipated by other means resulting in a load relaxation phenomenon, again a property of biological materials.

\section{Journal requirements}

Statement of principal findings.

Injuries for all musculoskeletal structures result from an inability to counter applied forces whilst the structure is lengthening. Injury during shortening would only occur if the unit had inadequate compressive strength, which we have demonstrated is prevented by anatomical constraints during normal operation.

\section{Strengths and weaknesses of the study}

The strengths of these deductions is it gives a comprehensive and easily understandable mechanism for all musculoskeletal injuries. The deductions are based in physical science giving validity to the deductions. The most significant weakness is the deductions are not able to be assessed with any experiment at this stage.

\section{Strengths and weaknesses in relation to other studies, discussing particularly any differences in results}

No other study as to our knowledge has deduced a mechanism for all musculoskeletal injuries.

Meaning of the study: possible mechanisms and implications for clinicians or policymakers.

This study gives us an understanding of the mechanism for all musculoskeletal injuries allowing us to assign an etiology to many musculoskeletal conditions which will be helpful in diagnosis, treatment and rehabilitation.

\section{Unanswered questions and future research}

This needs to be proven by experimentation as this becomes possible.

\section{Conflict of interests}

The Authors declare that they have no conflict of interests regarding the publication of this paper.

\section{References}

1. Handoll HH, Gillespie WJ, Gillespie LD, Madhok R. Moving towards evidence-based healthcare for musculoskeletal injuries: featuring the work of the Cochrane Bone, joint and Muscle Trauma Group. J R Soc Promot Health. 2007;127:168173.

2. Scott A, Docking S, Vicenzino B, et al. Sports and exercise-re- 
lated tendinopathies: a review of selected topical issues by participants of the second International Scientific Tendinopathy Symposium (ISTS) Vancouver. 2012. Br J Sports Med. 2013;47:536-544.

3. Freckleton G, Pizzari T. Risk factors for hamstring muscle strain injury in sport: a systematic review and meta-analysis. $\mathrm{Br}$ J Sports Med. 2013;47:351-358.

4. Lappin AK, Monroy JA, Pilarski JQ, et al. Recovery of elastic potential energy powers ballistic prey capture in toads. J Exper Biology. 2006;209:2535-2553.

5. Lindstedt SL, Reich TE, Keim P, La Stayo PC. Do muscles function as adaptable locomotor springs? J Exper Biology. 2002;205:2211-2216.

6. Monroy JA. Elastic properties of muscle during active shortening. Inter Comp Biol. 2006;46:e100.

7. Monroy JA, Lappin AK, Nishikawa KC. Elastic Properties of Active Muscle - On the rebound. Exer Sports Science Reviews. 2007;35:174-179.

8. Roberts TJ, Azizi E. Flexible mechanisms: the diverse roles of biological springs in vertebrate movement. J Exper Biology. 2011;214:353-361.

9. Azizi E, Robert TJ. Geared up to stretch: pennate muscle behavior during active lengthening. J Exper Biology. 2014 217:376-381.

10. Dickinson MH, Farley CT, Full RJ, et al. How animals move: an integrative view. Science. 2000;88:100-106.

11. Fukashiro S, Hay DC, Nagano A. Biomechanical behavior of muscle-tendon complex during dynamic human movements. J Appl Biomech. 2006;22:131-147.

12. Roberts TJ. The integrated function of muscles and tendons during locomotion. Comp Biochem Physiol A Mol Integr Physiol. 2002;133:1087-1099.

13. Sverdlova NS, Witzel U. Principles of determination and verification of muscle forces in the human musculoskeletal system muscle forces to minimise bending stress. J Biomech. 2010; 43:387-396.

14. Giancoli DC. Physics for Scientists \& Engineers with Modern Physics Volume 1, 4th edition. Pearson Education Limited, UK. 2014

15. Roberts TJ, Konow N. How tendons buffer energy dissipation by muscle. Exerc Sport Sci Rev. 2013;41:186-193.

16. Vogt M, Hoppeler HH. Eccentric exercise: mechanisms and effects when used as training regime or training adjunct. J Appl Physiol (1985). 2014;116:1446-1454.

17. Herbert RD, Héroux ME, Diong J, et al. Changes in the length and three-dimensional orientation of muscle fascicles and aponeuroses with passive length changes in human gastroc- nemius muscles. J Physiol. 2015;593:441-455.

18. Konow N, Azizi E, Roberts TJ. Muscle power attenuation by tendon during energy dissipation. Proc Biol Sci. 2012;279: 1108-1113.

19. Huxley $\mathrm{H}, \mathrm{Hanson} \mathrm{J}$. Changes in the cross-striations of muscle during contraction and stretch and their structural interpretation. Nature. 1954;173:973-976.

20. Raspanti M, Manelli A, Franchi M, Ruggeri A. The 3D structure of crimps in the rat Achilles tendon. Matrix Biol. 2005;24:503507.

21. Magnusson SP, Marici MV, Maganaris CN, Kjaer M. Human tendon behavious and adaptation, in vivo. J Physiol. 2008; 586:71-81.

22. Kwansa AL, Freeman JW. Elastic energy storage in an unmineralized collagen type I molecular model with explicit solvation and water infiltration. J Theor Biol. 2010;21:691-697.

23. Bohm S, Mersmann F, Tettke M, et al. Human Achilles tendon plasticity in response to cyclic strain: effect of rate and duration J Exper Biology. 2014;217:4010-4017.

24. Wolff J. The Law of Bone Remodeling. Berlin Heidelberg New York: Springer, 1986 (translation of the German 1892 edition).

25. Reeves ND, Marici MV, Maganaris CN. Strength training alters the viscoelastic properties of tendons in elderly humans. Muscle Nerve. 2003;48:74-81.

26. Padulo J, Oliva F, Frizziero A, Maffulli N. Muscles, Ligaments and Tendons Journal. Basic Principles and recommendations in clinical and field science research: 2016 update. MLTJ. 2016;6(1):1-5.

27. Christensen RM. Defining Yield Stress and Failure Stress (Strength) in The Theory of Materials Failure. Ed. R. Christensen, Oxford University Press, UK. 2013.

28. Dolman B, Verrall GM, Reid I. Physical principles demonstrate that the biceps femoris muscle relative to the other hamstring muscles exerts the most force: implications for hamstring muscle strain injuries. Muscles Ligaments Tendons Journal. 2014;17:371-377.

29. La Croix AS, Duenwald-Kuehl SE, Lakes RS, Vanderby R Jr. Relationship between tendon stiffness and failure: a metaanalysis. J App Physiol (1985). 2013;115:43-51.

30. Mammone JF, Hudson SM. Micromechanics of bone strength and fracture. J Biomech. 1993;26:439-446.

31. Rho JY, Ashman RB, Turner CH. Young's modulus of trabecular and cortical bone material: ultrasonic and microtensile measurements. J Biomech. 1993;26:111-119.

32. Linari $M$, Brunello $E$, Reconditi $M$, et al. Force generation by skeletal muscle is controlled by mechanosensing in myosin filaments. Nature. 2015;528:276-279. 